\title{
The bimodal jitter: a single fibre electromyographic finding
}

\author{
BARBARA THIELE ${ }^{1}$ AND ERIK STÅLBERG
}

From the Department of Clinical Neurophysiology, University Hospital, Uppsala, Sweden

SYNOPSIS The jitter, obtained by SFEMG recordings, mainly reflects the neuromuscular transmission time variability and is usually Gaussian distributed. Here is reported the bimodal distribution of the interpotential intervals, the flip-flop phenomenon. Long flip-flop in cases of reinnervation may indicate alternatively continuous and saltatory nerve conduction. Short flip-flop in normal subjects may be due to muscle fibre interaction or nerve conduction irregularities.

The muscle fibres of a motor unit are distributed randomly over a certain area in the muscle (Edström and Kugelberg, 1968), measuring less than $10 \mathrm{~mm}$ in diameter (Buchthal et al., 1957).

1 Fellow of the Deutsche Forschungsgemeinschaft.
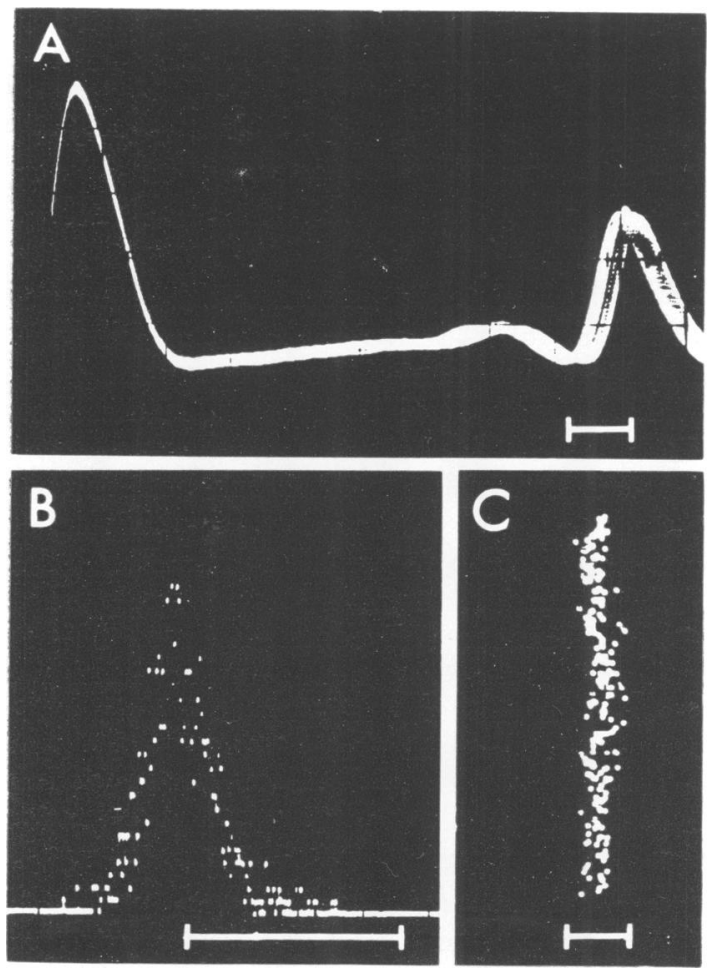

An electrode of the type used in single fibre electromyography (SFEMG), with leading-off surfaces $25 \mu$ in diameter, inserted at random in the muscle usually records activity from one (in $70 \%$ ) or sometimes two muscle fibres $(25 \%)$ belonging to the same motor unit at one recording site (Stålberg and Ekstedt, 1973). When activity from two muscle fibres belonging to the same motor unit is recorded, there is a variability in the time interval between the action potentials at consecutive discharges, the jitter, which is mainly due to a variability in the neuromuscular delay. This is normally of the order of $5-50 \mu \mathrm{s}$ expressed as mean consecutive difference (MCD) of the interval between the potentials (Stålberg et al., 1971). The distribution of the time intervals between the potentials is mainly Gaussian (Ekstedt et al., 1974) (Fig. 1).

In cases where reinnervation occurs, there is an increased fibre density of the motor units and therefore activity from up to 10 muscle fibres in

FIG. 1. Normal jitter in a potential pair. A. Original recording, 10 sweeps superimposed. The oscilloscope sweep is triggered on the fast positive-negative deflection of the first action potential. Its initial part is therefore not seen. The second potential appears with slight variability in relation to the first, the jitter. $\mathrm{B}$. Nonsequential histogram of interpotential intervals. Note unimodal distribution. C. Oscilloscope sweeps continuously moved downwards. Jitter seen as a variability around a certain mean value of interpotential intervals. Bar: $200 \mu s$. 

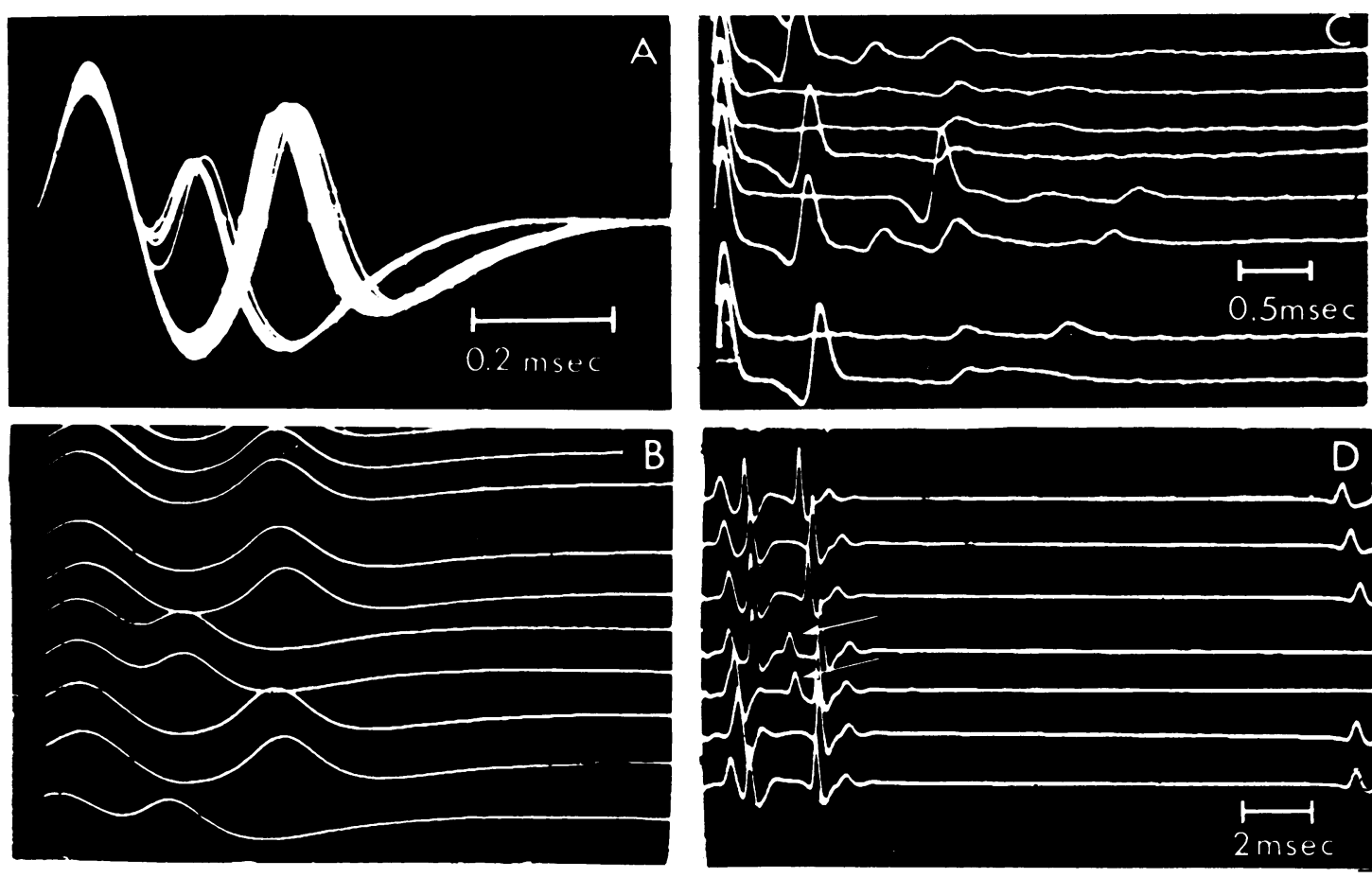

FIG. 2. Examples of bimodal jitter in different neuromuscular disorders. A and $\mathrm{B}$ : the same potential pair froro a patient with myasthenia gravis. In A, 20 sweeps are superimposed. In B the oscilloscope sweep is continuous? moved downwards. The latency change is $150 \mu \mathrm{s}$. C. Muscular dystrophy. Increased jitter. Latency chang\&. $\overrightarrow{0}$ $1 \mathrm{~ms}$. Note that there are occasional blockings of the jumping potential. D. Progressive spinal musculä료 atrophy. The last spike component in the complex occasionally appears $16 \mathrm{~ms}$ earlier (arrows).

one motor unit is recorded. The compound action potential may have a duration of up to 45 ms. Many of the spike components in the complex show an increased jitter in relation to each other due to a variability in the motor end-plates and also in the transmission time in the newly formed sprouts. Sometimes during sustained muscular activity, dropping out of action potentials is seen as a result of transmission block in some of these structures. The pathologically increased jitter is also mainly Gaussian--that is, the time intervals between the triggering spike and each of the other spike components are unimodally distributed around a certain mean value with a smaller or larger variability, small or large jitter.

Sometimes, however, it is observed that one or more of the action potentials in a double or polyspike recording show a bimodal distribution of the interspike intervals at consecutive discharges. The time interval between the triggering and another action potential in the complex has two mean values around both of which there is a certain variability (Fig. 2). The phenomenon is here called 'flip-flop'. It is found both in compound action potentials occurring in reinnervated muscle and in potential pairs recorded in healthy muscles. In this paper a description of the phenomenon and comments upon its possible causes are given. The investigation was focused on the large flip-flop in reinnervation $\mathrm{O}$ complexes.

\section{METHODS}

In two normal subjects, four subjects with partial N reinnervation after repeated electromyographic (EMG) needle insertions and 23 patients with neuro- 


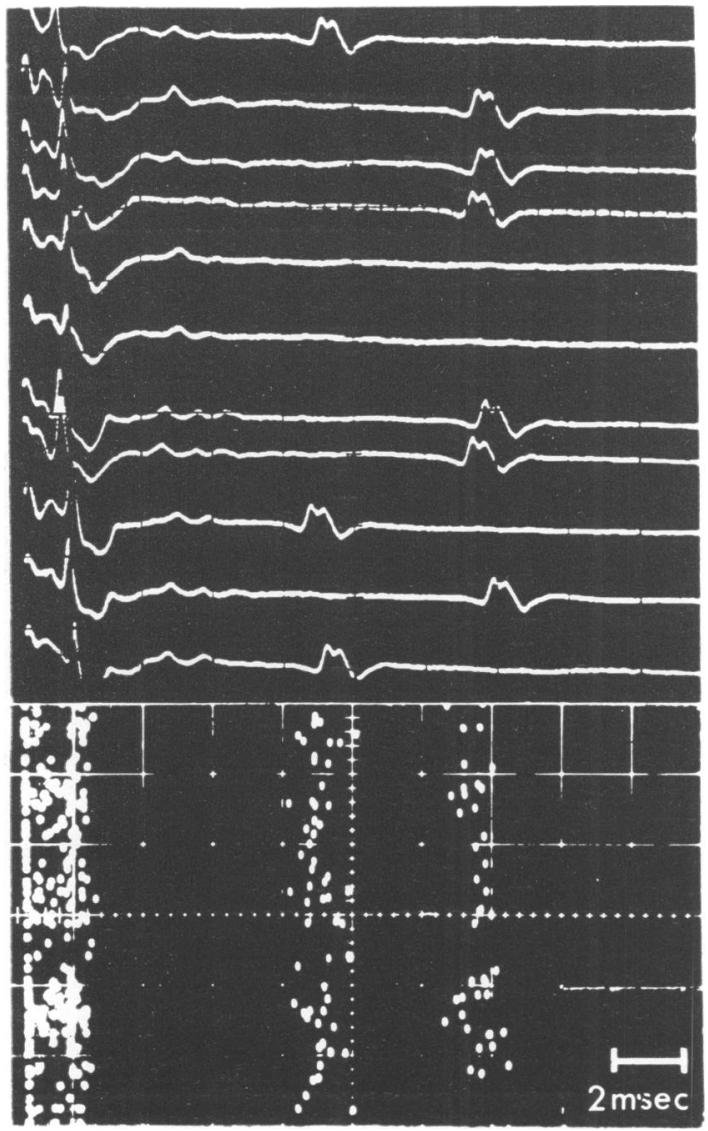

FIG. 3. Flip-flop in reinnervation compound action potential. The jumping potentials are late components of the recorded complex and generated from two muscle fibres. The sweep continuously moved downwards. Upper part. Original recordings. Note occasional missing of the jumping potential pair. Lower part. The different parts of the complex are represented by dots. The jitter in the jumping potentials is grossly equal in the two different positions.

muscular disorders SFEMG was performed for the study of the flip-flop phenomenon in the $\mathrm{m}$. extensor digitorum communis or in the $\mathrm{m}$. biceps brachii during slight voluntary contraction. Moreover, the phenomenon has been noticed many times in normal and diseased muscles but then not especially studied.

Two types of electrodes were used; one with two leading off surfaces, each $25 \mu$ in diameter, one with 14 leading off surfaces arranged $2+12$ in two parallel rows along the axis of the needle. The latter arrangement made it possible to measure the propagation velocity and to determine the propagation direction of the action potential along the muscle fibre when passing the recording electrode (Stålberg, 1966).

The action potentials were displayed on an oscilloscope (Tektronix 565) and recorded on a tape recorder (Akai X 330). Analysis was made on a storage oscilloscope (Tektronix 549). Time measurements were made with a time interval counter (HP $5245 \mathrm{~L}$ ) and recorded in digital form on a magnetic tape (Dymec 2546). The calculations were made on a CDC 3600 computer. The analysis included calculation of the jitter (MCD) separately in both positions for the jumping potential where this was possible - that is, usually meaning that the mean intervals between the two latencies differed by more than $200 \mu \mathrm{s}$. The interpotential intervals were plotted as sequential or nonsequential histograms.

In a few cases it was possible to study the flip-flop phenomenon during different discharge rates, during intravenous injection of d-tubocurarine, edrophonium (Tensilon), and during ischaemia.

\section{RESULTS}

According to earlier experiences the flip-flop phenomenon is usually found in one out of 20 recorded potential pairs in normal subjects. The phenomenon was seen somewhat more frequently in the patients in this investigation. In compound motor unit action potentials one or sometimes two paired spike components (Fig. 2) jumped between two different positions in relation to the other spikes in the complex. The 'jumping' action potential was of identical shape and amplitude in both positions. Usually one of the two latencies in the bimodal distribution was preferred, in some experiments the shorter, in some the longer latency. The probability of appearance in one or the other position was not determined by the position in the preceding discharges. The results presented in the Table can be divided into two parts; one with jumps shorter than $250 \mu$ s and one with longer jumps (Fig. 3). These longer jumps are seen less frequently than the short-that is, less than one out of 20 recordings. In the recordings with short jumps (100-250 $\mu \mathrm{s})$ found both in normal subjects, and in muscles with reinnervation the jitter was normal in the two positions and grossly equal, and the mean interval between the reference (triggering) and jumping action potential ranged between 50 and $800 \mu$ s. The larger jumps (300 $\mu \mathrm{s}-16 \mathrm{~ms})$ were never found in 
TABLE

RESULTS

\begin{tabular}{|c|c|c|c|c|c|c|c|}
\hline \multirow[t]{3}{*}{ Diagnosis } & \multicolumn{2}{|c|}{ Flip-flop } & \multirow{2}{*}{\multicolumn{2}{|c|}{$\begin{array}{l}\text { Shortest } \\
\text { latency }\end{array}$}} & \multicolumn{2}{|c|}{ Jitter } & \multirow[t]{3}{*}{ Comments } \\
\hline & \multirow{2}{*}{ (msec) } & \multirow{2}{*}{$(\mu s)$} & & & \multirow{2}{*}{$\begin{array}{l}\text { Short } \\
\text { latency }\end{array}$} & \multirow{2}{*}{$\begin{array}{l}\text { Long } \\
\text { latency }\end{array}$} & \\
\hline & & & (msec) & $(\mu s)$ & & & \\
\hline PMA* & 16 & & 3 & & Increased & - & - \\
\hline PMA & $15 \cdot 5$ & & 2 & & - & - & - \\
\hline PMA & 12 & & 10 & & Increased & Equal & - \\
\hline PMA & $10 \cdot 1$ & & $1 \cdot 2$ & & $160 \mu s$ & $190 \mu \mathrm{s}$ & - \\
\hline PMA & 9 & & 32 & & - & - & - \\
\hline PMA & 5 & & 8 & & Increased & $\begin{array}{c}\text { Somewhat } \\
\text { greater }\end{array}$ & $\begin{array}{l}\text { Paired flip-flop with occasional } \\
\text { blockings }\end{array}$ \\
\hline ALS $\dagger$ & 4 & & 5 & & 一 & - & Occasional blockings \\
\hline Intramuscular nerve trauma & 4 & & 2 & & $130 \mu \mathrm{s}$ & $110 \mu \mathrm{s}$ & $\begin{array}{l}\text { Injection of d-tubocurarine and } \\
\text { edrophonium }\end{array}$ \\
\hline ALS & $3 \cdot 7$ & & 7 & & $300 \mu \mathrm{s}$ & $300 \mu \mathrm{s}$ & - \\
\hline PMA & 3 & & 4 & & Increased & $\begin{array}{l}\text { Somewhat } \\
\text { greater }\end{array}$ & Paired flip-flop \\
\hline Muscular dystrophy & \multicolumn{2}{|l|}{$\begin{array}{l}1 \\
1 \cdot 5\end{array}$} & \multicolumn{2}{|l|}{$\begin{array}{l}1 \cdot 2 \\
2 \cdot 2\end{array}$} & Increased & Equal & 3 modal distribution \\
\hline \multirow{4}{*}{$\begin{array}{l}\text { ALS } \\
\text { Distal hereditary myopathy } \\
\text { Distal hereditary myopathy } \\
\text { ALS }\end{array}$} & \multicolumn{2}{|l|}{1} & \multicolumn{2}{|l|}{0.5} & $80 \mu \mathrm{s}$ & 一 & - \\
\hline & & 700 & \multicolumn{2}{|l|}{$2 \cdot 2$} & Increased & 一 & Occasional blockings \\
\hline & & 700 & \multicolumn{2}{|l|}{2} & Increased & Equal & - \\
\hline & & $\begin{array}{l}600 \\
600\end{array}$ & \multicolumn{2}{|l|}{$\begin{array}{l}100 \\
700\end{array}$} & Increased & Equal & 3 modal jitter \\
\hline \multicolumn{2}{|l|}{ Distal hereditary myopathy } & 600 & \multicolumn{2}{|l|}{1.6} & Increased & Equal & - \\
\hline Paramyotonia & & 500 & $2 \cdot 6$ & & & & Often blockings \\
\hline ALS & & 500 & \multicolumn{2}{|r|}{500} & Increased & Equal & - \\
\hline \multirow{2}{*}{$\begin{array}{l}\text { Myasthenia gravis } \\
\text { PMA }\end{array}$} & & 500 & \multicolumn{2}{|r|}{350} & Increased & Equal & - \\
\hline & & 300 & \multicolumn{2}{|r|}{400} & Increased & $\begin{array}{c}\text { Somewhat } \\
\text { greater }\end{array}$ & - \\
\hline Intramuscular nerve trauma & & 300 & & 900 & Increased & - & Studied during hyperventilation \\
\hline \multirow{4}{*}{$\begin{array}{l}\text { Normal, intramuscular nerve } \\
\text { trauma, myasthenia gravis, } \\
\text { PMA, ALS, paramyotonia, } \\
\text { distal hereditary myopathy }\end{array}$} & \multicolumn{2}{|c|}{$\begin{array}{l}\text { Range } \\
100-250 \mu s\end{array}$} & \multicolumn{2}{|c|}{$\begin{array}{l}\text { Range } \\
50-800 \mu s\end{array}$} & Normal & $\begin{array}{l}\text { Equal } \\
\text { within } \\
\pm 10 \mu \mathrm{s}\end{array}$ & \\
\hline & Mear & 148 & Mea & 276 & & & \\
\hline & SD & 45 & & 176 & & & \\
\hline & $\mathbf{n}$ & 30 & $\mathbf{n}$ & 30 & & & \\
\hline
\end{tabular}

* PMA = Progressive spinal muscular atrophy.

† ALS = Amyotrophic lateral sclerosis.

normal muscles, only in pathologically changed muscles where more or less pronounced SFEMG signs of reinnervation were found (even in the investigated myopathies and in the patient with myasthenia gravis). The jitter was always increased and only slightly different in the two positions. When different, the jitter was a little greater in the late position, with one exception where it was slightly greater in the early position. Sometimes blocking occurred in the jumping potential. The jumping potentials were typically late components. The short latency between the triggering and the jumping potential was usually more than $500 \mu \mathrm{s}$. In two recordings with flipflop in reinnervation complexes two or three action potentials were jumping together. There was a normal jitter between these jumping potentials. Together they had an increased jitter in relation to the rest of the action potential com- plex. In one of these recordings the jumping potentials were missing due to neurogenic block (Fig. 3) (Stålberg and Thiele, 1972).

DISCHARGE RATE Experiments were made on both long and short jumps. The subjects were asked to keep a certain pre-set discharge rate of a motor unit with the aid of a ratemeter. Recording was made for different levels between 8 and $15 \mathrm{imp} / \mathrm{s}$ during 500 discharges. The jumping potential did not change the frequency of appearance in any of the two positions with changing discharge rate.

In another type of experiment the subjects were asked to make the discharge rate irregular. Analysis was made concerning the influence of the interval to the preceding discharge upon the position of the jumping potential. The interval did not influence the preferred position. 
EDROPHONIUM AND D-TUBOCURARINE Five eXperiments were performed. In one recording with a short flip-flop $3 \mathrm{mg}$ d-tubocurarine was injected intravenously. The jitter increased by the same amount in both positions from about 20-30 $\mu \mathrm{s}$ to 70-90 $\mu \mathrm{s}$. No blocking occurred in the jumping potential. The frequency of appearance in one position changed from about $10 \%$ to about $20 \%$. This change was, however, not significantly larger than the spontaneous fluctuation in appearance frequency in one position seen before the injection.

In another experiment recording was made from a compound action potential, a reinnervation complex, with a long flip-flop (4 ms). Seventy seconds after the injection of $4 \mathrm{mg} \mathrm{d}$ tubocurarine one spike component changed its preferred position from a short latency $(2 \mathrm{~ms})$ to a longer $(6 \mathrm{~ms})$. After $10 \mathrm{mg}$ edrophonium intravenously the shorter latency was preferred again.

In two experiments on recordings with long flip-flop and in one with short flip-flop $10 \mathrm{mg}$ edrophonium was injected intravenously. No definite change in the jumping could be observed.

PROPAGATION VELOCITY ALONG MUSCLE FIBRE The propagation velocity of the muscle fibre action potential could be measured accurately only in recordings with long jumps. In short jumps there was interference with the triggering potential making measurements invalid. Three experiments of this kind were performed. The propagation velocity was the same for the action potential at short and long latency and the propagation direction of the impulse was the same in both positions.

ISCHAEMIA The forearm was made ischaemic by a blood pressure cuff around the upper arm. SFEMG recording was made in $\mathrm{m}$. extensor digitorum communis before and during ischaemia.

In one experiment with short flip-flop the potential changed its preferred position after three minutes of ischaemia but after about six minutes the potential appeared more frequently in the initial position again. After five minutes of ischaemia the potential showed an increased jitter in both positions and after seven minutes there was a total block (Dahlbäck et al., 1970).

In two other experiments with ischaemia, while recording a jitter with unimodal distribution, the jitter increased and changed to a bimodal mode with short flip-flop and before total block was again unimodal, now around the new latency.

\section{DISCUSSION}

As seen in the Table, the material could be divided into two separate parts: one type of recording with normal jitter and with latency changes shorter than $250 \mu \mathrm{s}$, found both in normal subjects and in patients with neuromuscular disorders: another type with increased jitter and with larger jumps, occurring only in potentials found in muscles with signs of reinnervation. These two types of flip-flop recordings may have different causes and are discussed separately.

\section{LONG FLIP-FLOP IN REINNERVATION}

As the phenomenon of flip-flop involved only one or two spike components in an action potential complex generated from muscle fibres belonging to the same motor unit it can at once be localized peripherally in the motor unit. The shape, amplitude and propagation velocity of the action potential was the same in both positions and the jumping potential never appeared simultaneously at the two latencies. Thus, the jumping potential cannot represent activity from two different muscle fibres but is generated by one muscle fibre. With regard to the magnitude of the latency changes, it is very unlikely that the phenomenon takes place in the muscle fibre itself-for example, as an effect of a sudden change of propagation velocity of the muscle fibre action potential. The cause is most likely to be found in the motor end-plates or in the peripheral nerve tree.

Could the two latencies be due to impulse initiation in two motor end-plates on the same muscle fibre, one of them partially blocking? If recording were done from a double innervated muscle fibre only action potentials initiated in the motor end-plate nearest to the electrode were recorded. If the transmission in this motor endplate should happen to block, the action poten- 
tial from the other one will be unmasked and recorded with an increased latency corresponding to the transmission time between the two motor end-plates. The formation of double motor end-plates on one muscle fibre innervated by sprouts originating from the same motor nerve fibre is described as taking place during reinnervation (Coërs and Woolf, 1959). In such a case the jitter would usually differ in the two positions, as each motor end-plate has its typical jitter (Stålberg et al., 1971). As a matter of fact, the jitter was sometimes unequal in the two positions, usually somewhat larger in the late position, but no great differences were obtained. The finding that curare changed the position of the potential from an early to a late latency, an effect reversed by edrophonium, might support the possibility of two motor end-plates as might the two experiments where the flip-flop appeared during ischaemia when the jitter was increased. Ischaemia is, however, known to change the nerve excitability (Gilliatt and Willison, 1963) and therefore other explanations of these results are also possible (vide infra).

However, at least in the recordings where two spike components jumped together, the flip-flop cannot be explained by double motor end-plates. The fact that the two jumping potentials also jittered in relation to each other indicates that they are derived from two different muscle fibres and not from one split fibre (Ekstedt and Stålberg, 1969b). Here the cause must be localized in the terminal nerve tree. This may hold also for flipflop in single spikes, but the neurogenic localization could be shown only where at least four muscle fibres were recorded-that is, paired jumping in relation to two components. In a triple recording a jump in the triggering potential could not be differentiated from a jump in the two other components. There were no principal differences concerning jitter, frequency, and length of the jumps between the recording with single spike and double spike jumping. The results with changed preferred position of the jumping potential after d-tubocurarine and edrophonium do not necessarily exclude the neural theory in single flip-flop, as curare (Standaert, 1964) and edrophonium (Okamoto and Riker, 1969) are both shown to have an effect also on the nerve membrane.

As known from histological studies (Young,
1942; Wohlfart, 1957) the newly formed sprouts in reinnervation are not at all or only insufficiently myelinated. They are usually thin, elongated, and take an irregular course to the muscle fibres, sometimes even forming loops (Woolf, 1969). Thus, there are anatomical prerequisites for an uncertain impulse transmission in the nerve twigs. Some histological changes in the nerve twigs which might possibly be responsible for the sudden shortening and lengthening of the conduction time will be discussed.

Ephaptic transmission If close anatomical contact takes place between two parts of the same nerve twig due to 'looping' or between two nerve twigs in the same motor unit or between one muscle fibre and one nerve twig in the same motor unit, an ephaptic transmission might take place. In this way there may be a sudden shortening of the impulse transmission time to the muscle fibre under investigation. Of the three possibilities for ephaptic transmission mentioned above, the muscle fibre-nerve fibre cross

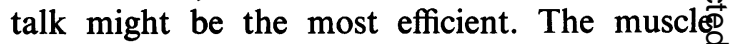
fibre is a stronger and larger generator than 2 nerve fibre and furthermore contact between $\frac{}{0}$ muscle fibre and nerve fibre in the same motoo unit is probably more common than nerve-nerve $\overrightarrow{0}$ contact. Under normal conditions, ephaptiç transmission is very unlikely, as the activity in one nerve fibre gives only slight changes in excitability of an adjacent nerve fibre (Katz and Schmitt, 1940). An action potential is not initiated unless the membrane potential is near the trigger level (Jasper and Monnier, 1938) or extreme experimental conditions are set up (Arvanitaki, 1942). In cases of reinnervation, however, the nerve twigs are formed of thin unmyelinated sprouts of which the excitability is unknown. There are electromyographic signs of hyperexcitability in nerve or muscle fibre (increased insertion activity) and clinical signs of spontaneous nerve activity (tendency to muscle cramps). In experiments with $\mathrm{C}$-fibres (Hallin and Torebjörk, 1970) it has been shown that the naked portion of the nerve, the receptor part, is considerably more sensitive to electrical stimulation than the other part of the nerve fibre which is unmyelinated but surrounded by Schwann's cells. Thus hyperexcitability in the newly formed and uninsulated sprouts might offer possibilities 
for ephaptic transmission. On the other hand, conduction velocity of the impulse in the nerve twigs is low (long duration of the reinnervation potentials where the jumping potential is a late component), which normally parallels a low excitability. Furthermore, the threshold to electrical stimulation in peripheral nerve is increased in cases with demyelinating nerve lesions (Wright and McQuillen, 1973). If this holds true also for the reinnervation sprouts the flip-flop phenomenon is very unlikely to be caused by ephaptic transmission.

Uncertain impulse transmission along nerve The newly formed sprouts are first unmyelinated (Wohlfart, 1957) and there is an impaired impulse transmission (Stålberg and Thiele, 1972). During the maturation process the sprouts will become myelinated, initially uninterrupted without nodes of Ranvier which appear at a later stage (Vizoso and Young, 1948). In neurophysiological terms this corresponds to an increase in the nerve conduction velocity and in the safety factor for the impulse transmission. Consequently, the duration of the action potentials complexes decreases, the jitter normalizes and there are no longer any blockings.

During this myelination process there may be, hypothetically, a transitional stage where the impulse could sometimes be conducted continuously over a part of the sprout, sometimes saltatory due to critical isolation properties of the growing myelin sheath, giving rise to the long flip-flop. This explanation seems for the moment to be the most likely. As the flip-flop phenomenon is seen relatively seldom in reinnervation potentials, it is assumed that the transitional stage of alternation between continuous and saltatory conduction respectively is of short duration.

SHORT FLIP-FLOP IN RECORDINGS WITH NORMAL JITTER Many times previously in normal subjects and during the investigation of flip-flop in reinnervation complexes this phenomenon was seen in action potential pairs with normal jitter. The cause of this small flip-flop has not been studied completely in this investigation but some comments will be made.

The small flip-flop seems not to be correlated with any neuromuscular disorder. Recordings in a normal muscle are only exceptionally made from more than three muscle fibres and in these a simultaneous jump of two spikes has not been observed. Thus, there is no explicit evidence for a neurogenic localization in short flip-flop.

As an explanation of the double distributed jitter, Ekstedt (1964) assumed two motor endplates on one muscle fibre. This seems unlikely because it implies that one of these motor endplates blocks now and then as discussed on $p$. 407. Blocking of action potentials is, however, never seen in other recordings with a normal jitter but appears when the jitter is above $70-100 \mu \mathrm{s}$ regardless of the reason for the increase (Ekstedt and Stålberg, 1967; Ekstedt and Stålberg, 1969a; Stålberg and Ekstedt, 1973).

One experiment with curare showed a jitter increase of the same order in both positions and a slightly changed frequency of appearance in the two positions. No blockings occurred. The effects were too small to allow any conclusions to be drawn. Thus, there was no evidence for the two motor end-plate hypothesis.

Another theoretical possibility could be a sudden change in propagation velocity in one of the muscle fibres. An interaction between two adjacent nerve fibres with a change in propagation velocity is described by Katz and Schmitt (1940) as happening in certain circumstances (the propagation velocity should not differ by more than $10 \%$ and the impulse should be roughly synchronous). In our recordings, the muscle fibres with a small flip-flop were lying close to each other as the amplitude of the two potentials was usually of the same order of magnitude, waxing and waning mainly in parallel when the recording electrode was moved to and fro. Moreover, the potentials usually appeared over the recording electrode with a short interval and were thus almost simultaneously in time within $800 \mu \mathrm{s}$ usually $300 \mu \mathrm{s}$. If an optimal situation randomly existed for interaction between the muscle fibres this would cause a changed propagation velocity in some discharges, and the interval between the two recorded potentials could show two different means.

A third possibility may also be a localization of the phenomenon to the terminal nerve twig. Could the impulse jump over two internodes, missing, for example, one which is shorter than 
normal, an anatomical finding reported by Vizoso and Young (1948)? At an internodal distance of $1 \mathrm{~mm}$ a conduction velocity of $5-10 \mathrm{~m} / \mathrm{s}$ in the periphery should give a $100-200 \mu s$ shortening of the conduction time, corresponding to the observed short flip-flop.

In normal muscles the explanation for the flip-flop is for the moment uncertain. However, when performing SFEMG and measuring the jitter it is of importance to be aware of the phenomenon. In these recordings the jitter is bimodal or at least shows negative excess and when calculated in the ordinary way as MCD an abnormally high value will be found without blockings. This must not be mistaken for a potential pair with increased jitter.

\section{SUMMARY}

A single fibre EMG investigation was made in muscles with reinnervation of different aetiology. In recordings from two or more muscle fibres from one motor unit, the variability in interpotential interval between one action potential and the rest of the action potential complexes at consecutive discharges, the jitter, was bimodal instead of unimodal which is the normal finding. This phenomenon was called flip-flop. It occurred in recordings with increased jitter and the flip-flop could measure up to $16 \mathrm{~ms}$. Experiments with d-tubocurarine, edrophonium, and ischaemia were made. Possible explanations for the phenomenon were discussed such as double motor end-plates, ephaptic transmission between two peripheral parts of the same motor unit, and continuous or saltatory conduction alternatively in remyelinating nerve twigs. The last interpretation seemed most likely. Another type of flipflop with short jumps was seen in normal action potential pairs with normal jitter in normal and pathological muscles. The pathophysiological mechanisms were discussed and are probably different from those in the cases with reinnervation. In cases of small flip-flop the possibility could not be excluded that the phenomenon took place in the muscle fibre itself as an effect of interaction between two adjacent muscle fibres from the same motor unit, but sudden irregularities in the saltatory nerve conduction could also be a possibility.
The investigation was supported by the Swedish Medical Research Council, Grant No. 04X-135. The skilful technical assistance of Miss Y. Markestad and Engineer G. Lovén, who also made the needle electrodes is gratefully acknowledged.

\section{REFERENCES}

Arvanitaki, A. (1942). Effects evoked in an axon by the activity of a contiguous one. Journal of Neurophysiology, 5 , 89-108.

Buchthal, F., Guld, Ch., and Rosenfalck, P. (1957). Multielectrode study of the territory of a motor unit. Acta Physiologica Scandinavica, 39, 83-103.

Coërs, C., and Woolf, A. L. (1959). The Innervation of Muscle. Blackwell: Oxford.

Dahlbäck, L. O., Ekstedt, J., and Stålberg, E. (1970). Ischemic effects on impulse transmission to muscle fibers in man. Electroencephalography and Clinical Neurophysiology, 29, 579-591.

Edström, L., and Kugelberg, E. (1968). Histochemical composition, distribution of fibres and fatiguability of single motor units. Anterior tibial muscle of the rat. Journal of Neurology, Neurosurgery, and Psychiatry, 31, 424-433.

Ekstedt, J. (1964). Human single muscle fiber action potentials. Acta Physiologica Scandinavica, 61, Suppl. 226.

Ekstedt, J., and Stålberg, E. (1967). Myasthenia gravis. Diagnostic aspects by a new electrophysiological method Opuscula Medica, 12, 73-76.

Ekstedt, J., and Stålberg, E. (1969a). The effect of non paralytic doses of D-tubocurarine on individual moton end-plates in man, studied with a new electrophysiologica method. Electroencephalography and Clinical Neuro physiology, 27, 557-562.

Ekstedt, J., and Stålberg, E. (1969b). Abnormal connection between skeletal muscle fibers. Electroencephalography an $\bar{f}$ Clinical Neurophysiology, 27, 607-609.

Ekstedt, J., Nilsson, G., and Stålberg, E. (1974). Calculation of the electromyographic jitter. Journal of Neurology, Neurosurgery, and Psychiatry. (In press).

Gilliatt, R. W., and Willison, R. G. (1963). The refractory and supernormal periods of the human median nerve. Journal of Neurology, Neurosurgery, and Psychiatry, 26, 136-147.

Hallin, R. G., and Torebjörk, H. E. (1970). Afferent and efferent $\mathrm{C}$ units recorded from human skin nerves in situ. A preliminary report. Acta Societatis Medicorum Upsaliensis, 75, 277-281.

Jasper, H. H., and Monnier, A. M. (1938). Transmission of excitation between excised non-myelinated nerves. An artificial synapse. Journal of Cellular and Comparative Physiology, 11, 259-277.

Katz, B., and Schmitt, O. H. (1940). Electric interaction between two adjacent nerve fibres. Journal of Physiology, 97, 471-488.

Okamoto, M., and Riker, W. F., Jr. (1969). Subacute denervation: a means of disclosing mammalian motor nerve terminals as critical sites of acetylcholine and facilitatory drug action. Journal of Pharmacology and Experimental Therapeutics, 166, 217-224.

Stålberg, E. (1966). Propagation velocity in human muscle fibers in situ. Acta Physiologica Scandinavica, 70, Suppl. 287.

Stålberg, E., and Ekstedt, J. (1973). Single fibre EMG and microphysiology of the motor unit in normal and diseased human muscle. In New Developments in Electromyography and Clinical Neurophysiology, pp. 113-129. Karger: Basel.

Stålberg, E., Ekstedt, J., and Broman, A. (1971). The electromyographic jitter in normal human muscles. 
Electroencephalography and Clinical Neurophysiology, 31, 429-438.

Stålberg, E., and Thiele, B. (1972). Transmission block in terminal nerve twigs. A single fibre electromyographic finding in man. Journal of Neurology, Neurosurgery, and Psychiatry, 35, 52-59.

Standaert, F. G. (1964). The action of d-tubocurarine on the motor nerve terminal. Journal of Pharmacology and Experimental Therapeutics, 143, 181-186.

Visozo, A. D., and Young, J. Z. (1948). Internodal length and fibre diameter in developing and regenerating nerves. Journal of Anatomy, 82, 110-134.
Wohlfart, G. (1957). Collateral regeneration from residual motor nerve fibers in amyotrophic lateral sclerosis. Neurology (Minneap.), 7, 124-134.

Woolf, A. L. (1969). Pathological anatomy of the intramuscular nerve ending. In Disorders of Voluntary Muscle. 2nd edn., pp. 203-237. Edited by J. N. Walton. Churchill: London.

Wright, E. A., and McQuillen, M. P. (1973). Hypoexcitability of ulnar nerve in patients with normal motor nerve conduction velocities. Neurology (Minneap.), 23, 78-83.

Young, J. Z. (1942). The functional repair of nervous tissue. Physiological Reviews, 22, 318-374. 